\title{
A periodic map for linear barycentric rational trigonometric interpolation
}

\author{
Jean-Paul Berrut, Giacomo Elefante* \\ Department of Mathematics, University of Fribourg, Pérolles, Fribourg 1700, Switzerland
}

Keywords:

Barycentric rational interpolation

Trigonometric interpolation

Conformal maps

\begin{abstract}
A B S T R A C T
Consider the set of equidistant nodes in $[0,2 \pi)$,

$$
\theta_{k}:=k \cdot \frac{2 \pi}{n}, \quad k=0, \ldots, n-1 .
$$

For an arbitrary $2 \pi$-periodic function $f(\theta)$, the barycentric formula for the corresponding trigonometric interpolant between the $\theta_{k}$ 's is

$$
T[f](\theta)=\frac{\sum_{k=0}^{n-1}(-1)^{k} \operatorname{cst}\left(\frac{\theta-\theta_{k}}{2}\right) f\left(\theta_{k}\right)}{\sum_{k=0}^{n-1}(-1)^{k} \operatorname{cst}\left(\frac{\theta-\theta_{k}}{2}\right)},
$$

where $\operatorname{cst}(\cdot):=\operatorname{ctg}(\cdot)$ if the number of nodes $n$ is even, and $\operatorname{cst}(\cdot):=\csc (\cdot)$ if $n$ is odd. Baltensperger [3] has shown that the corresponding barycentric rational trigonometric interpolant given by the right-hand side of the above equation for arbitrary nodes introduced in [9] converges exponentially toward $f$ when the nodes are the images of the $\theta_{k}$ 's under a periodic conformal map. In the present work, we introduce a simple periodic conformal map which accumulates nodes in the neighborhood of an arbitrarily located front, as well as its extension to several fronts. Despite its simplicity, this map allows for a very accurate approximation of smooth periodic functions with steep gradients.
\end{abstract}

\section{Introduction}

The polynomial interpolating between Chebyshev nodes of the second kind is a very efficient way of approximating smooth functions on a finite interval. It converges exponentially rapidly for analytic functions, faster than algebraically (spectrally) for infinitely smooth ones and with an order corresponding to that of differentiability for several times differentiable functions. The MATLAB-based software Chebfun as well as some (pseudo)spectral methods for partial differential equations are based on it $[23,24]$.

The barycentric formula for its evaluation reads

$$
P_{n}(x)=\frac{\sum_{k=0}^{n^{\prime \prime}} \frac{(-1)^{k}}{x-x_{k}} F\left(x_{k}\right)}{\sum_{k=0}^{n^{\prime \prime}} \frac{(-1)^{k}}{x-x_{k}}},
$$

\footnotetext{
* Corresponding author.

E-mail addresses: jean-paul.berrut@unifr.ch (J.-P. Berrut), giacomo.elefante@unifr.ch (G. Elefante).
} 
where the $x_{k}$ are the interpolation points (nodes), $F$ is the interpolated function and the double prime means that the first and the last term of the sum are multiplied by $\frac{1}{2}$. For a description of the barycentric representation, the reader may consult [13].

In [9], the first author has suggested the use of (1) also when the interpolation points $x_{k}$ are not the Chebyshev ones. The result is a rational function with no poles on $\mathbb{R}$ [9], which we shall denote by $r_{n}[f]$. Baltensperger et al. [6] have shown that $r_{n}[f]$ retains the exponential convergence for analytic functions, when the $x_{k}$ 's are images of the Chebyshev nodes under a conformal map. This may be used to improve the stability of pseudospectral methods in time evolution problems [5] and to diminish the number of iterations in the solution of the systems of equations arising from pseudospectral methods for stationary problems [10]. But it may also be used to concentrate the nodes in the vicinity of fronts in order to improve accuracy [11], a property which has led to several applications, starting with the solution of stationary problems with fronts in [12] and of time evolution problems in [22]. The authors of the latter paper have introduced a map which moves (known) singularities of the function away from their domain. This permitted the accurate solution of explosion problems in [22] and of infiltration problems in [14], for instance.

The work [11] makes use of the Bayliss-Turkel map [7], which involves two parameters for describing the front, one for its location, the other for its strength, and optimizes the parameters as functions of $f$, rendering $r_{n}[f]$ nonlinear in $f$, with spectacular results; see [4] for a close alternative. [11] also presents a method for extending the map to functions with several fronts, which has been applied recently $[18,20]$ and will be used in the present paper as well.

Letting $x=\cos \theta, x_{k}=\cos \theta_{k}$ in (1) shows that the latter is the cosine polynomial interpolant of the $2 \pi$-periodic even function $F(\cos \phi)$ [8] between the equidistant nodes

$$
\theta_{k}:=k \cdot \frac{2 \pi}{n}, \quad k=0, \ldots, n-1
$$

For an arbitrary $2 \pi$-periodic function $f(\theta)$, the corresponding trigonometric interpolant between the equidistant $\theta_{k}$ from (2) reads [16]

$$
T[f](\theta)=\frac{\sum_{k=0}^{n-1}(-1)^{k} \operatorname{cst}\left(\frac{\theta-\theta_{k}}{2}\right) f\left(\theta_{k}\right)}{\sum_{k=0}^{n-1}(-1)^{k} \operatorname{cst}\left(\frac{\theta-\theta_{k}}{2}\right)},
$$

where $\operatorname{cst}(\cdot)=\operatorname{ctg}(\cdot)$ if the number of nodes $n$ is even, and $\operatorname{cst}(\cdot)=\csc (\cdot)$ if $n$ is odd.

An extension of Chebfun to periodic functions using trigonometric interpolation has recently been constructed by Wright and al. [25].

In [9], the first author has suggested to use $T[f](\phi)$ as in formula (3) for arbitrary nodes $\phi_{k}$ with

$$
0 \leq \phi_{0}<\phi_{1}<\cdots<\cdots<\phi_{n-1}<2 \pi
$$

and proved that the corresponding linear rational trigonometric interpolant does not have any poles on $[0,2 \pi)$ for any set of nodes satisfying (4).

Baltensperger [3] has later shown that, similarly to the rational interpolant (1), (3) converges exponentially toward $f$ when the nodes are the images of the $\theta_{k}$ 's from (2) under a periodic conformal map. Baltensperger tested his results merely with a periodic version of the Tal-Ezer map, which improves the accuracy only for functions with a front in the center of the interval.

In his thesis [21], Tee has constructed a periodic map which pushes (known) poles of $f$ away from the domain $[0,2 \pi$ ), but involves special functions.

In the present work, we introduce a simple periodic conformal map which accumulates nodes in the neighborhood of an arbitrarily located front, as well as its extension to several fronts. Despite its simplicity, this map allows for a very accurate approximation of smooth periodic functions with steep gradients.

\section{Exponential convergence of the interpolant}

As mentioned before, Baltensperger [3] was able to extend the theorem for the exponential convergence of $r_{n}[f]$ to the case of trigonometric interpolation. His result reads as follows.

Theorem 1. Let $g$ be a complex valued function with period $2 \pi$ such that $g([0,2 \pi])=[0,2 \pi]$ and such that

$$
w(\phi, \psi):=\frac{\operatorname{cst}((g(\phi)-g(\psi)) / 2)}{\operatorname{cst}((\phi-\psi) / 2)}
$$

is bounded and analytic in $S_{a_{1}} \times S_{a_{1}}$, where $S_{a_{1}}:=\left\{\zeta:|\operatorname{Im} \zeta| \leq a_{1}\right\}\left(a_{1}>0\right)$. Let $f$ be a function to be approximated such that $f_{\circ} g$ is a complex-valued function with period $2 \pi$.

Let $T[f](\phi):=T[f \circ g](\theta), \phi=g(\theta)$, be the rational function (3) interpolating $f$ between the transformed points $\phi_{k}=g\left(\theta_{k}\right)$ with $\theta_{k}$ as in (2). Then, for every $\phi \in[0,2 \pi]$, we have the following:

(i) If $f \circ g$ has simple jump discontinuities in the $p$ th derivative, then

$$
|f(\phi)-T[f](\phi)|=\mathcal{O}\left(n^{-p}\right) ;
$$


(ii) If $f \circ g$ is analytic in a strip $S_{a_{2}}:=\left\{\zeta:|\operatorname{Im} \zeta| \leq a_{2}\right\}$, where $a_{1} \geq a_{2} \geq 0$ and $|f(\phi)| \leq M$, then

$$
|f(\phi)-T[f](\phi)|=\mathcal{O}\left(\rho^{-n}\right), \quad \text { with } \rho:=e^{a_{2}} .
$$

One can therefore use conformal maps to move equidistant nodes and still retain rapid convergence.

One idea is to use the maps for polynomial interpolation appropriately modified in order to make them periodic [3]. For instance, the map by Kosloff and Tal-Ezer [19]

$$
g(x)=\arcsin (\alpha x) / \arcsin (\alpha), \quad 0<\alpha<1,
$$

becomes [3]

$$
g(\theta)=2 \arccos \left(\frac{\arcsin (\alpha \cos (\theta / 2))}{\arcsin (\alpha)}\right), \quad 0<\alpha<1,
$$

while the one of Bayliss and Turkel [7]

$$
g(x)=\beta+\frac{1}{\alpha} \tan (\lambda(x-\mu))
$$

becomes

$$
g(\theta)=2 \arccos \left(\beta+\frac{1}{\alpha} \tan (\lambda(\cos (\theta / 2)-\mu))\right)
$$

with the following parameters $\mu$ and $\lambda$ :

$$
\lambda=\frac{\gamma+\eta}{2}, \quad \mu=\frac{\gamma-\eta}{\gamma+\eta}, \quad \gamma=\arctan (\alpha(1+\beta)), \quad \eta=\arctan (\alpha(1-\beta)) .
$$

We shall use these two maps for comparison in the numerical examples; for other maps for the non-periodic case, see, e.g. $[2,18,22]$.

\section{A periodic conformal map}

We shall now introduce the conformal maps which constitute the central part of this work. For $z \in \mathbb{C}$, consider the Möbius transformation [15]

$$
h_{a}(z)=\frac{z+a}{1+z \bar{a}}, \quad \text { with }|a|<1 .
$$

$h_{a}$ maps the unit disk onto itself in such a way that the image of the point $-a$ is mapped to 0 . Recall that, since $h_{a}$ is a Möbius transformation, it maps (arcs of) circles to (arcs of) circles. Notice also that the inverse function of $h_{a}$ is $h_{-a}$. Moreover, $h_{a}$ maps connected subsets of $\mathbb{C}$ conformally and leaves the unit circle $E$ invariant, i.e., maps it onto itself. The image of the boundary point $e^{i \theta}$ is

$$
e^{i \psi(\theta)}:=h_{a}\left(e^{i \theta}\right)=\frac{e^{i \theta}+a}{1+e^{i \theta} \bar{a}} .
$$

$\psi(\theta)$ is the so-called boundary correspondence function of the map $h_{a}$ from the unit disk onto itself [17]; it will be the basis of our changes of variable.

Let us consider $a$ real; if we write $e^{i \theta}=x+i y$, we have $x^{2}+y^{2}=1$ and we can write $e^{i \psi(\theta)}$ as

$$
\frac{e^{i \theta}+a}{1+e^{i \theta} a}=\frac{x+i y+a}{1+(x+i y) a}=\frac{x+2 a+a^{2} x}{1+2 a x+a^{2}}+i \frac{y-a^{2} y}{1+2 a x+a^{2}}=u_{a}(x, y)+i v_{a}(x, y) \text {. }
$$

The partial derivative of $u_{a}(x, y)$ with respect to $a$ is

$$
\frac{\partial u_{a}(x, y)}{\partial a}=\frac{\left(x^{2}-1\right)\left(2 a^{2}-2\right)}{\left(1+2 a x+a^{2}\right)^{2}}=-\frac{y^{2}\left(2 a^{2}-2\right)}{\left(1+2 a x+a^{2}\right)^{2}}
$$

and is positive when $0<a<1$, which means that all points of $E$ but \pm 1 move to the right. Therefore, many elements of the set $\left\{e^{i \theta}\right\}$, where $\boldsymbol{\theta}:=\left\{\theta_{k}=\frac{2 k \pi}{n}, k=0, \ldots, n-1\right\}$ is the set of the equidistant nodes (2), will cluster around 1 (see Fig. 1 ).

When we consider a complex value of $a$ such as $a=\rho e^{i \varphi}, \varphi \in(0,2 \pi)$, many images of $\left\{e^{i \theta}\right\}$ will cluster around $e^{i \varphi}$, since this general case is the rotation of the previous case by the angle $\varphi$. So, if we restrict ourselves to the boundary correspondence function, the arguments of the mapped points $h_{a}\left(e^{i \theta}\right)$ will cluster around $\varphi$. 


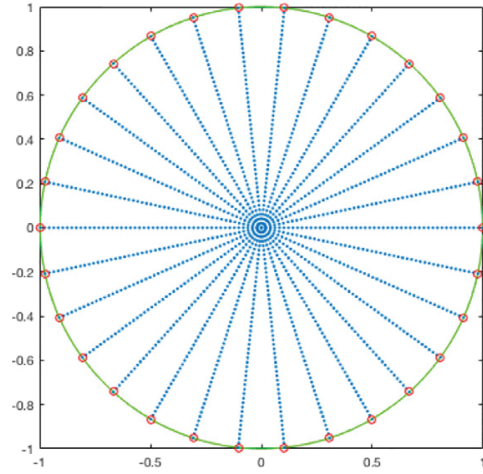

(a) $\rho=0$

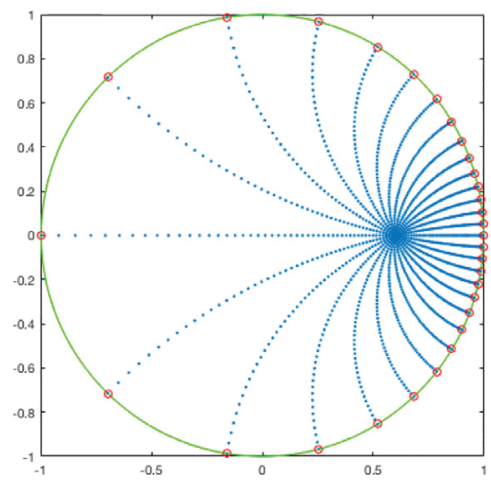

(c) $\rho=0.6$

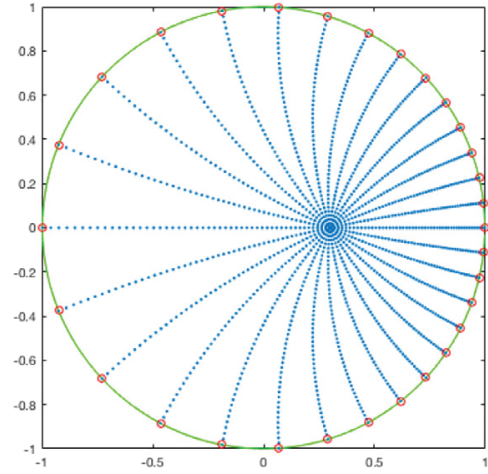

(b) $\rho=0.3$

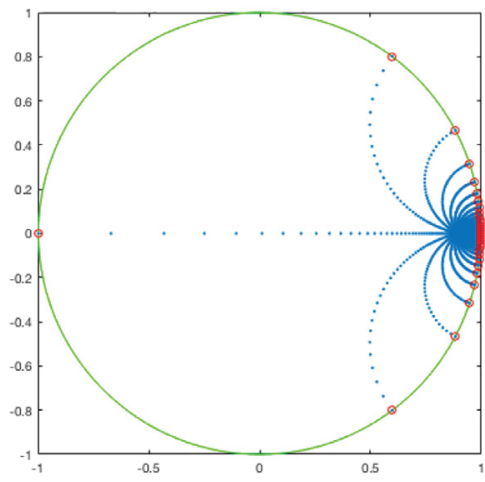

(d) $\rho=0.9$

Fig. 1. Uniform points on the circle and their radii mapped with $h_{a}$ with various values of $a=\rho$.

\subsection{A map for a single front}

Our map $g$, then, will be the boundary correspondence function, which we obtain by taking the logarithm with value in $[0,2 \pi)$ of $(10)$ :

$$
g_{a}(\theta)=-i \log \left(\frac{e^{i \theta}+a}{1+e^{i \theta} \bar{a}}\right)
$$

(since the principal value of the logarithm lies in $(-\pi, \pi]$, we must choose another branch).

In general we have $g_{a}(0) \neq 0$, in which case, since the function is periodic, we consider the function $\widetilde{g}_{a}(\phi):=g_{a}(\phi+\gamma)$ for a constant $\gamma$ such that $\widetilde{g}_{a}(0)=0$.

We can now use these new points to interpolate a function with the barycentric trigonometric interpolant and take advantage of Theorem 1. In the present work, we wish to construct an exponentially convergent approximant of a function $f$ of which we know that it has a front at $\theta=\varphi$. We do this by choosing a $\rho$ in $a=\rho e^{i \varphi}$ such that the interpolation points $\boldsymbol{\theta}$ cluster around $\varphi$ and applying the barycentric rational interpolant (3) for $\phi:=g_{a}(\theta)$ instead of $\theta$.

\subsection{Multiple fronts}

In case the function $f$ has several fronts, we need to construct a conformal map that accumulates the points around all the locations of the fronts. As proposed in [12], we consider the inverse functions $g_{q}^{-1}$ with parameters $\rho_{q}, \varphi_{q}$, for $q=1, \ldots, J$, one $g_{q}$ for each front, and we define the map $\bar{g}^{-1}$ as

$$
\bar{g}^{-1}(\phi)=\frac{1}{J} \sum_{q=1}^{J} g_{q}^{-1}(\phi) ;
$$

$\bar{g}$ will cluster the nodes around the various fronts. Since the latter lie at the (known) points $\varphi_{q}$, we want to choose $\rho_{q}$ such that $a_{q}=\rho_{q} e^{i \varphi_{q}}$ are adequate parameters in $\bar{g}(\theta)$. 
We have

$$
\begin{aligned}
& \frac{1}{J} \sum_{q=1}^{J}-i \log \left(\frac{e^{i \phi}-a_{q}}{1-e^{i \phi} \overline{a_{k}}}\right)=\theta \\
& \prod_{k=1}^{J}\left(\frac{e^{i \phi}-a_{k}}{1-e^{i \phi} \overline{a_{k}}}\right)=e^{j i \theta},
\end{aligned}
$$

or

$$
\prod_{k=1}^{J}\left(e^{i \phi}-a_{k}\right)-e^{j i \theta} \prod_{k=1}^{J}\left(1-e^{i \phi} \overline{a_{k}}\right)=0 .
$$

Setting $z:=e^{i \phi}$, we have to find the zero of a polynomial of degree less than or equal to $J$ for every value of $\phi$ at which we need that of $\theta$, in particular for each of the $n$ interpolation points.

However, in the case of two fronts we can compute the inverse function analytically. Indeed, let us consider $a=\rho_{1} e^{i \varphi_{1}}$ and $b=\rho_{2} e^{i \varphi_{2}}$, the parameters of the functions $g_{1}^{-1}$ and $g_{2}^{-1}$, respectively. (13) becomes

$$
\left(\frac{e^{i \phi}-a}{1-e^{i \phi} \bar{a}}\right)\left(\frac{e^{i \phi}-b}{1-e^{i \phi} \bar{b}}\right)=e^{2 i \theta}
$$

or

$$
\frac{\left(e^{i \phi}-a\right)\left(e^{i \phi}-b\right)}{1+e^{2 i \phi} \bar{a} \bar{b}-e^{i \phi}(\bar{a}+\bar{b})}=e^{2 i \theta},
$$

which leads to the equation

$$
e^{2 i \phi}\left(1-e^{2 i \theta} \bar{a} \bar{b}\right)+e^{i \phi}\left(e^{2 i \theta}(\bar{a}+\bar{b})-(a+b)\right)+a b-e^{2 i \theta}=0
$$

for $\phi$. Setting $z:=e^{i \phi}$, this turns into

$$
\begin{aligned}
z^{2}\left(1-e^{2 i \theta} \bar{a} \bar{b}\right)+z\left(e^{2 i \theta}(\bar{a}+\bar{b})-(a+b)\right)+a b-e^{2 i \theta} \\
=z^{2}\left(e^{-i \theta}-e^{i \theta} \bar{a} \bar{b}\right)+z\left(e^{i \theta}(\bar{a}+\bar{b})-e^{-i \theta}(a+b)\right)+e^{-i \theta} a b-e^{i \theta} \\
=z^{2}\left(e^{-i \theta}-e^{i \theta} \bar{a} \bar{b}\right)-2 i z \operatorname{Im}\left(e^{-i \theta}(a+b)\right)+e^{-i \theta} a b-e^{i \theta}=0 .
\end{aligned}
$$

The reduced discriminant of this second degree equation for $z$ is

$$
\begin{aligned}
\frac{\Delta}{4} & =\left(i \operatorname{Im}\left(e^{-i \theta}(a+b)\right)\right)^{2}+\left(e^{-i \theta}-e^{i \theta} \bar{a} \bar{b}\right)\left(e^{i \theta}-e^{-i \theta} a b\right) \\
& =\left|e^{i \theta}-e^{-i \theta} a b\right|^{2}-\operatorname{Im}\left(e^{-i \theta}(a+b)\right)^{2}
\end{aligned}
$$

and the solutions are

$$
z_{1,2}(\theta)=\frac{i \operatorname{Im}\left(e^{-i \theta}(a+b)\right) \pm\left(\frac{\Delta}{4}\right)^{1 / 2}}{e^{-i \theta}-e^{i \theta} \bar{a} \bar{b}} .
$$

But

$$
z_{1}(\theta+\pi)=\frac{-i \operatorname{Im}\left(e^{-i \theta}(a+b)\right)+\left(\frac{\Delta}{4}\right)^{1 / 2}}{-e^{-i \theta}+e^{i \theta} \bar{a} \bar{b}}=\frac{i \operatorname{Im}\left(e^{-i \theta}(a+b)\right)-\left(\frac{\Delta}{4}\right)^{1 / 2}}{e^{-i \theta}-e^{i \theta} \bar{a} \bar{b}}=z_{2}(\theta),
$$

so that the solution is unique up to a translation; translating it to have the image of 0 in 0 , we have

$$
\phi=-i \log \left(z_{1}(\theta)\right) \text {. }
$$

One could also obtain analytical maps for the cases of three and four fronts, but this requires the use of more involved formulas such as that of Cardano for the third degree equation and even more complicated ones for the fourth degree equation; moreover, a stable implementation of such formulas requires care.

\section{Numerical examples}

\subsection{One front function}

We have first considered a function similar to that of Example 1 in [11], but modified it in order to make it periodic,

$$
f_{1}(\theta)=e^{1 /(\sin (\theta+\pi)+1.5)}+\cos (4(\sin (\theta+\pi)+0.5))+\frac{\operatorname{erf}(\delta(\sin (\theta+\pi)+1))}{\operatorname{erf}(\delta)}
$$




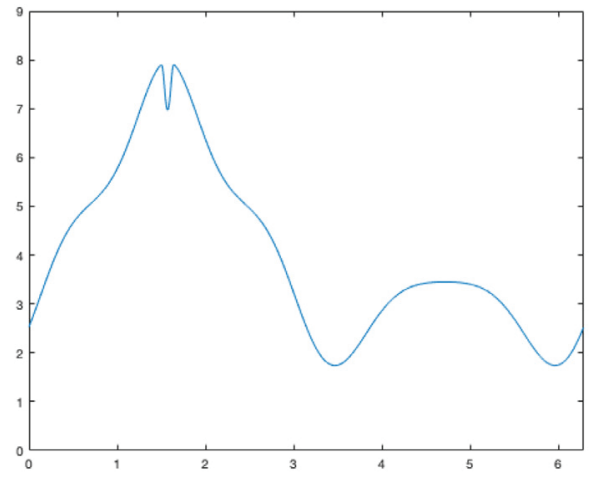

(a) Function $f_{1}$ with $\epsilon=10^{6}$

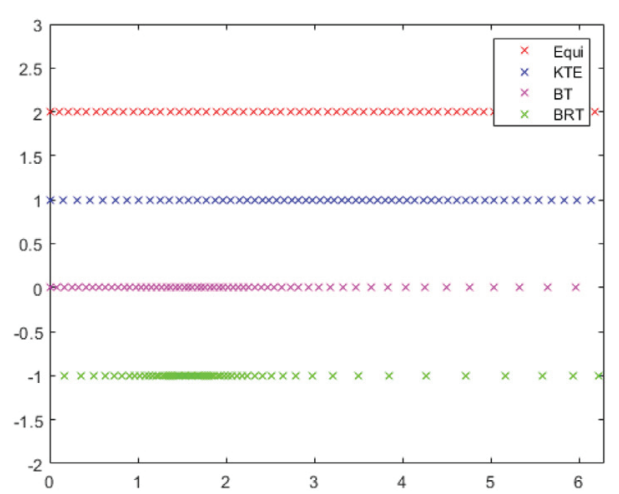

(b) 60 nodes using the various maps and the same parameters as in Table 2

Fig. 2. Left: an example with the function $f_{1}$; Right: various point sets.

Table 1

Error with the function $f_{1}$ with $\epsilon=10^{4}$.

\begin{tabular}{llllllll}
\hline$n$ & Equi & KT & & BT & & BRT & \\
\hline 10 & $5.7201 \mathrm{e}-01$ & $5.7295 \mathrm{e}-01$ & $(0.05)$ & $3.7049 \mathrm{e}-01$ & $(3.05)$ & $4.5603 \mathrm{e}-01$ & $(0.04)$ \\
20 & $4.3166 \mathrm{e}-01$ & $4.3332 \mathrm{e}-01$ & $(0.05)$ & $5.0872 \mathrm{e}-01$ & $(11.30)$ & $3.5469 \mathrm{e}-02$ & $(0.40)$ \\
40 & $6.0408 \mathrm{e}-02$ & $5.0592 \mathrm{e}-02$ & $(0.99)$ & $6.0059 \mathrm{e}-02$ & $(2.05)$ & $2.0856 \mathrm{e}-03$ & $(0.46)$ \\
80 & $4.9557 \mathrm{e}-03$ & $5.0356 \mathrm{e}-03$ & $(0.05)$ & $1.3034 \mathrm{e}-02$ & $(2.05)$ & $5.8500 \mathrm{e}-07$ & $(0.51)$ \\
160 & $4.5164 \mathrm{e}-05$ & $4.2683 \mathrm{e}-05$ & $(0.86)$ & $3.1541 \mathrm{e}-03$ & $(2.05)$ & $1.9540 \mathrm{e}-14$ & $(0.54)$ \\
320 & $1.1680 \mathrm{e}-10$ & $1.1921 \mathrm{e}-10$ & $(0.47)$ & $7.8051 \mathrm{e}-04$ & $(2.05)$ & $1.7764 \mathrm{e}-14$ & $(0.62)$ \\
\hline
\end{tabular}

Table 2

Error with the function $f_{1}$ with $\epsilon=10^{6}$.

\begin{tabular}{llllllll}
\hline$n$ & Equi & KT & & BT & & BRT & \\
\hline 15 & $9.6326 \mathrm{e}-01$ & $5.9939 \mathrm{e}-01$ & $(0.92)$ & $4.8577 \mathrm{e}-01$ & $(3.60)$ & $4.6128 \mathrm{e}-01$ & $(0.52)$ \\
30 & $9.9971 \mathrm{e}-01$ & $5.1234 \mathrm{e}-01$ & $(0.59)$ & $4.2361 \mathrm{e}-01$ & $(3.05)$ & $2.0320 \mathrm{e}-01$ & $(0.56)$ \\
60 & $4.7472 \mathrm{e}-01$ & $4.6612 \mathrm{e}-01$ & $(0.94)$ & $5.7724 \mathrm{e}-02$ & $(3.05)$ & $3.9628 \mathrm{e}-03$ & $(0.63)$ \\
120 & $8.5163 \mathrm{e}-02$ & $7.9048 \mathrm{e}-02$ & $(0.94)$ & $9.1171 \mathrm{e}-03$ & $(2.75)$ & $5.2002 \mathrm{e}-06$ & $(0.68)$ \\
240 & $9.2787 \mathrm{e}-03$ & $8.0863 \mathrm{e}-03$ & $(0.94)$ & $1.4085 \mathrm{e}-03$ & $(2.05)$ & $1.2257 \mathrm{e}-13$ & $(0.70)$ \\
480 & $6.2903 \mathrm{e}-05$ & $5.4821 \mathrm{e}-05$ & $(0.20)$ & $3.4604 \mathrm{e}-04$ & $(2.05)$ & $4.1744 \mathrm{e}-14$ & $(0.51)$ \\
\hline
\end{tabular}

with $\delta=\sqrt{0.5 \epsilon}$ for a parameter $\epsilon$ which characterizes the steepness of $f_{1}$. Here we have computed with the two cases $\epsilon=10^{4}$ and $\epsilon=10^{6}$. The front of this function is in $\theta=\frac{\pi}{2}$, as may be seen in Fig. 2.

To estimate the error we considered 2000 uniformly spaced points in $[0,2 \pi]$ and we computed the maximum difference between the interpolant and the function in these points.

In Tables 1 and 2 we made a comparison using various kinds of points. The first set of points were $n$ equidistant points in $[0,2 \pi$ ). We used the map (6) derived from that of Kosloff and Tal-Ezer (KT in the table) and that of Bayliss and Turkel (8) (BT in the table): as parameter $\alpha$ we chose the best values (written in parentheses in the tables) through a trial and error method within the sets [0.05: 0.01: 0.99] and [2.05: 0.05: 15], respectively; as parameter $\beta$ in Bayliss and Turkel we took the value for which the map clusters the points at the front $\pi / 2$, i.e., $\beta=1 / \sqrt{2}$. Since in $[7,11]$ the results did not noticably depend on a perturbation of the parameter $\beta$ about the location of the front, we refrained from trying other values. For the last column we used the map (11) presented above (BRT-for "barycentric rational trigonometric"-in the tables), where we chose the best $\rho$ within the set [0: 0.01: 0.99]. (One could go further by estimating an optimal $\rho$, in the spirit of [7], or numerically optimize it, as in [11], but our aim here is only to demonstrate the effectiveness of the proposed map in some situations.) For the same reason as for $\beta$ above, we fixed $\rho$ as the location of the front.

The approximate squaring of the error when $N$ is doubled reflects the exponential decay of the error predicted by Theorem 1.

The reason why the map (8) does not lead to exponential convergence is that its construction does not provide conformality. Indeed, $g^{\prime}(0)=0$ in view of the factor $\sin (\theta / 2)$ in the inner derivative; and an analytic function with vanishing derivative is not conformal [1, p. 133]. 


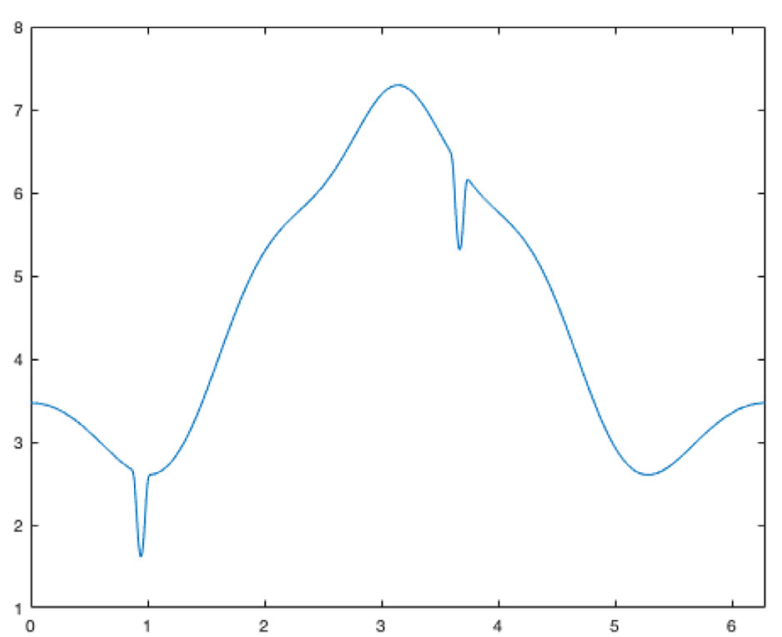

(a) The function $f_{2}$

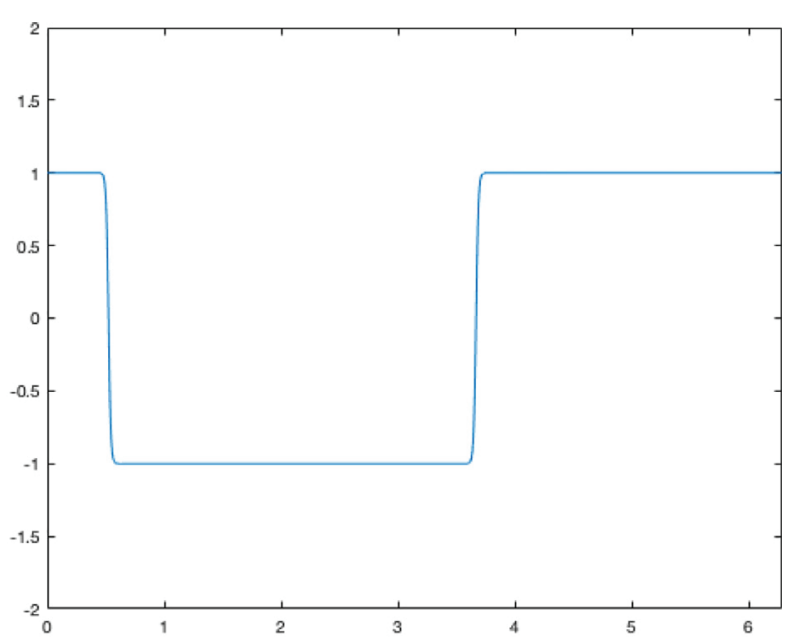

(b) The function $f_{3}$

Fig. 3. The two test functions.

Table 3

Error with the function $f_{2}$.

\begin{tabular}{llllll}
\hline$n$ & Equi & BT & & BRT & \\
\hline 60 & $4.7774 \mathrm{e}-01$ & $3.6941 \mathrm{e}-02$ & $(13.3)$ & $2.1843 \mathrm{e}-02$ & $(0.77)$ \\
120 & $8.6081 \mathrm{e}-02$ & $5.3467 \mathrm{e}-03$ & $(12.7)$ & $9.8636 \mathrm{e}-05$ & $(0.79)$ \\
240 & $9.3970 \mathrm{e}-03$ & $5.7084 \mathrm{e}-04$ & $(6.00)$ & $1.5925 \mathrm{e}-09$ & $(0.79)$ \\
480 & $6.3819 \mathrm{e}-05$ & $2.7993 \mathrm{e}-05$ & $(2.05)$ & $6.6613 \mathrm{e}-14$ & $(0.74)$
\end{tabular}

Table 4

Error with the function $f_{3}$.

\begin{tabular}{llllll}
\hline$n$ & Equi & BT & & BRT & \\
\hline 35 & $1.1156 \mathrm{e}+00$ & $3.0810 \mathrm{e}-01$ & $(12.35)$ & $1.9638 \mathrm{e}-02$ & $(0.90)$ \\
70 & $7.3624 \mathrm{e}-01$ & $1.0701 \mathrm{e}-01$ & $(13.70)$ & $1.0129 \mathrm{e}-03$ & $(0.87)$ \\
140 & $1.9318 \mathrm{e}-01$ & $8.6162 \mathrm{e}-03$ & $(14.65)$ & $2.0570 \mathrm{e}-06$ & $(0.86)$ \\
280 & $2.2589 \mathrm{e}-02$ & $2.1048 \mathrm{e}-04$ & $(10.10)$ & $1.9059 \mathrm{e}-12$ & $(0.85)$ \\
560 & $2.8214 \mathrm{e}-04$ & $3.0953 \mathrm{e}-05$ & $(2.80)$ & $9.7700 \mathrm{e}-15$ & $(0.89)$ \\
\hline
\end{tabular}

\subsection{Two fronts function}

As an example of a function with two fronts we have considered a modification of $f_{1}$ :

$$
f_{2}(\theta)=e^{1 /\left(\sin \left(\theta+\frac{\pi}{2}\right)+1.6\right)}+\cos \left(\pi\left(\sin \left(\theta+\frac{\pi}{2}\right)+0.5\right)\right)+\frac{\operatorname{erf}\left(\delta\left(\sin \left(\theta+\frac{\pi}{3}\right)+1\right)\right)}{\operatorname{erf}(\delta)}+\frac{\operatorname{erf}\left(\delta\left(\sin \left(\theta+\frac{6 \pi}{5}\right)+1\right)\right)}{\operatorname{erf}(\delta)},
$$

with $\delta=\sqrt{0.5 \epsilon}$, and $\epsilon=10^{6}$; this function has one front in $\theta_{1}=\frac{7}{6} \pi$ and another in $\theta_{2}=\frac{3}{10} \pi$.

The function

$$
f_{3}(\theta)=\tanh \left(50 \cos \left(\theta+\frac{\pi}{3}\right)\right),
$$

has two fronts as well, respectively in $\theta_{1}=\frac{\pi}{6}$ and $\theta_{2}=\frac{7}{6} \pi$.

The test functions $f_{2}$ and $f_{3}$ are plotted in Fig. 3.

As for $f_{1}$, we performed a trial an error search for the parameter of the map (16), in the same set as for $f_{1}$ and setting the same $\rho$ for both fronts, as the same parameter $\delta$ is used in the two erf-functions in $f_{2}$ and the same value 50 multiplies the two zero values of the cosine in $f_{3}$.

In Tables 3 and 4, we display a comparison with the equidistant nodes and with the periodic Bayliss and Turkel map with the same value of the parameter $\alpha$ for the two fronts, chosen with a trial and error in the same set as for $f_{1}$. Again, the $\beta$-values are the cosines of half the abscissae of the fronts. Since we do not have an explicit formula for the inverse of this map with multiple fronts, we approximated the $\phi_{k}$ by means of the function fsolve in MATLAB, i.e., as the values which solve the equation $\bar{g}^{-1}(\phi)=\theta$, where $\bar{g}^{-1}$ is the function in (12) with $J=2$ and $g_{q}^{-1}$ the inverse of the Bayliss and Turkel map. 


\section{Conclusions}

In this work we have introduced a periodic conformal map for shifting nodes on the interval of interpolation with the purpose of approximating periodic functions with fronts. Our experiments demonstrate that the suggested map indeed leads to exponentially convergent barycentric rational trigonometric interpolants, as to be expected from the theorem by Baltensperger. Such an exponential convergence is faster than any power of $1 / n$, in other words spectral. In the case of two fronts we have an analytical formula and do not need to numerically solve an equation for every point, as with other maps suggested in the literature. In many cases this leads to an enormous saving of computation. When more than two fronts are present, we merely have to solve a polynomial equation of corresponding degree for every node.

\section{Acknowledgement}

The authors thank Richard Baltensperger and a referee for their comments, which have improved the present work.

\section{References}

[1] L.V. Ahlfors, Complex Analysis: An Introduction of the Theory of Analytic Functions of one Complex Variable, Second edition, McGraw-Hill Book Co., New York-Toronto-London, 1966

[2] A. Alexandrescu, A. Bueno-Orovio, J.R. Salgueiro, Mapped Chebyshev pseudospectral method for the study of multiple scale phenomena, Comput. Phys. Commun. 180 (6) (2009) 912-919.

[3] R. Baltensperger, Some results on linear rational trigonometric interpolation, Comput. Math. Appl. 43 (2002) 737-746.

[4] R. Baltensperger, Barycentric rational interpolation with asymptotically monitored poles, Numer. Algorithms 57 (2011) 67-81.

[5] R. Baltensperger, J.-P. Berrut, The linear rational collocation method, J. Comput. Appl. Math. 134 (1-2) (2001) $243-258$.

[6] R. Baltensperger, J.-P. Berrut, B. Noël, Exponential convergence of a linear rational interpolant between transformed Chebyshev points, Math. Comput. 68 (227) (1999) 1109-1120.

[7] A. Bayliss, E. Turkel, Mappings and accuracy for Chebyshev pseudo-spectral approximations, J. Comput. Phys. 101 (2) (1992) 349-359.

[8] J.-P. Berrut, Baryzentrische Formeln zur trigonometrischen Interpolation. I, Z. Angew. Math. Phys. 35 (1) (1984) 91-105.

[9] J.-P. Berrut, Rational functions for guaranteed and experimentally well-conditioned global interpolation, Comput. Math. Appl. 15 (1) (1988) 1-16.

[10] J.-P. Berrut, R. Baltensperger, The linear rational pseudospectral method for boundary value problems, BIT 41 (5, suppl.) (2001) 868-879.

[11] J.-P. Berrut, H.D. Mittelmann, Adaptive point shifts in rational approximation with optimized denominator, J. Comput. Appl. Math. 164/165 (2004) $81-92$

[12] J.-P. Berrut, H.D. Mittelmann, Optimized point shifts and poles in the linear rational pseudospectral method for boundary value problems, J. Comput. Phys. 204 (1) (2005) 292-301.

[13] J.-P. Berrut, L.N. Trefethen, Barycentric Lagrange interpolation, SIAM Rev. 46 (3) (2004) 501-517.

[14] L. Cueto-Felgueroso, R. Juanes, Adaptive rational spectral methods for the linear stability analysis of nonlinear fourth-order problems, J. Comput. Phys. 228 (2009) 6536-6552.

[15] P. Henrici, Applied and Computational Complex Analysis, Vol. 1, Wiley-Interscience [John Wiley \& Sons], New York-London-Sydney, 1974.

[16] P. Henrici, Barycentric formulas for interpolating trigonometric polynomials and their conjugates, Numer. Math. 33 (2) (1979) $225-234$.

[17] P. Henrici, Applied and Computational Complex Analysis, Vol. 3, John Wiley \& Sons, Inc., New York, 1986.

[18] H.A. Jafari-Varzaneh, S.M. Hosseini, A new map for the Chebyshev pseudospectral solution of differential equations with large gradients, Numer. Algorithms 69 (1) (2015) 95-108.

[19] D. Kosloff, H. Tal-Ezer, A modified Chebyshev pseudospectral method with an $O\left(N^{-1}\right)$ time step restriction, J. Comput. Phys. 104 (2) (1993) $457-469$.

[20] E. Pindza, K. Patidar, E. Ngounda, Rational spectral collocation method for pricing American vanilla and butterfly spread options, in: Finite Difference Methods, Theory and Applications, in: Lecture Notes in Comput. Sci., 9045, Springer, Cham, 2015, pp. 323-331.

[21] T.W. Tee, An Adaptive Rational Spectral Method for Differential Equations with Rapidly Varying Solutions, PhD thesis, University of Oxford, 2006.

[22] T.W. Tee, L.N. Trefethen, A rational spectral collocation method with adaptively transformed Chebyshev grid points, SIAM J. Sci. Comput. 28 (2006) $1798-1811$.

[23] L.N. Trefethen, Spectral Methods in MATLAB, Society for Industrial and Applied Mathematics (SIAM), Philadelphia, 2000.

[24] L.N. Trefethen, Approximation Theory and Approximation Practice, SIAM, Philadelphia, 2013.

[25] G.B. Wright, M. Javed, H. Montanelli, L.N. Trefethen, Extension of Chebfun to periodic functions, SIAM J. Sci. Comput. 37 (5) (2015) C554-C573. 